\title{
Correction to: 'Getting it Right First Time': The Effects of Anxiety and Fear on a Birthing Woman
}

\author{
Helen Shallow
}

Correction to: K. Gutteridge (ed.), Understanding Anxiety, Worry and Fear in Childbearing, https://doi.org/10.1007/978-3-030-21063-2_9

This chapter was inadvertently published with incorrect affiliation for this chapter author. It has been corrected in this updated version.

Helen Shallow

Midwife, Writer and Presenter

Louth, England

The updated online version of this chapter can be found at https://doi.org/ 10.1007/978-3-030-21063-2_9 